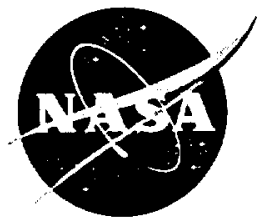

\title{
A Moving Grid Capability for NPARC
}

John W. Slater

Lewis Research Center, Cleveland, Ohio

Prepared for the

36th Aerospace Sciences Meeting and Exhibit

sponsored by the American Institute of Aeronautics and Astronautics

Reno, Nevada, January 12-15, 1998

National Aeronautics and

Space Administration

Lewis Research Center 


\section{Acknowledgments}

The author would like to thank Dr. Joongkee Chung of the Institute for Computational Methods in Propulsion (ICOMP) at the Ohio Aerospace Institute and Scott Townsend of NYMA, Inc., for their work in the development of NPARC v3.1. Further, the author would like to thank Charles Lawrence, Rich Blech, and Gary Cole of NASA Lewis for their support for the development of NPARC v3.1.

Available from

NASA Center for Aerospace Information 7121 Standard Drive

Hanover, MD 21076

Price Code: A03
National Technical Information Service 5287 Port Royal Road Springfield, VA 22100 Price Code: A03 


\title{
A MOVING GRID CAPABILITY FOR NPARC
}

\author{
John W. Slater* \\ NASA Lewis Research Center, Cleveland, Ohio
}

\begin{abstract}
Version 3.1 of the NPARC computational fluid dynamics flow solver introduces a capability to solve unsteady flow on moving multi-block, structured grids with nominally second-order time accuracy. The grid motion is due to segments of the boundary grid that translate and rotate in a rigid-body manner or deform. The grid is regenerated at each time step to accommodate the boundary grid motion. The flow equations and computational models sense the moving grid through the grid velocities, which are computed from a time-difference of the grids at two consecutive time levels. For three-dimensional flow domains, it is assumed that the grid retains a planar character with respect to one coordinate. The application and accuracy of NPARC v3.1 is demonstrated for flow about a flying wedge, rotating flap, a collapsing bump in a duct, and the unstart / restart flow in a variable-geometry inlet. The results compare well with analytic and experimental results.
\end{abstract}

\section{Introduction}

Version $3.1^{1}$ of the NPARC ${ }^{2,3}$ computational fluid dynamics (CFD) flow solver was released in OCtober 1997 with modifications that enhance the capability to solve unsteady flows and introduce a capability to simulate flows with moving, multi-block, structured grids. This paper discusses those modifications, explains the approach for defining the moving grid problem, and presents several test cases that demonstrate the application and accuracy of NPARC v3.1 for moving grids.

Moving grids in CFD arise when there is motion of a region of the flow domain relative to the rest of the flow domain. An example is a flow domain about a high-speed, variable-geometry, axisymmetric inlet. The centerbody of the inlet may translate

-Aerospace Engineer, Inlet Branch, AIAA Senior Member and collapse as part of the restart operation for the inlet once it has unstarted. The grid block containing the centerbody may deform to accommodate the centerbody motion. Such grid motion is considered moderate in that the magnitude of the motion is less than the scale of the block dimensions and the topology of the block remains intact.

NPARC v3.1 assumes that there are moderate levels of grid motion associated with the motion of segments of the boundary grid relative to the rest of the grid of the block. This motion may be a rigidbody translation and / or rotation about a point or a deformation of the segment according to a coded relation. The remainder of the grid of the block deforms to accommodate the boundary motion. This requires that some regions of the grid be regenerated at each time step. Efficiency in the grid regeneration process is obtained by limiting the regeneration to only those regions in which there is grid motion. Thus, the grid becomes a computed function of time. The flow equations and boundary conditions are expressed in terms of an absolute frame of reference with the grid motion accounted for through the grid velocities. The velocity of each grid point can be calculated from a time difference of the grids at two consecutive time levels. For three-dimensional flow domains, the grid is assumed to be "quasi-2d" or axisymmetric in which the grid consists of planar grids with respect to the "l"-coordinate.

The flow equations and physical models are first presented in integral form for a deforming control volume to show the influence of the velocities of the control surface. The finite-difference approximations are then discussed to show how the grid velocities influence the computational methods. The approach for defining the moving grid problem within NPARC v3.1 is then discussed. Several test cases involving moving grids are presented to demonstrate the application and accuracy of the moving grid capability. A few simple test cases involving supersonic flow over a stationary wedge, a flying wedge, and a rotating flap on a flat plate allow comparison with steadystate oblique shock theory. A test case involving the collapse of an axisymmetric bump in an annu- 
lar duct allow comparison with unsteady experiment data. The simulation of the unstart / restart operation of the NASA variable diameter centerbody (VDC) inlet provides a more complex application.

\section{Flow Equations}

The governing equations of the fluid flow are the Reynolds-averaged Navier-Stokes equations. To account for moving grids, the flow equations are written in an absolute frame of reference with the allowance that the control volume may deform in time. The integral form of the equations allowing for deformation can be expressed as

$$
\hat{Q}_{t}+\hat{R}=\hat{S}
$$

where $t$ is time and

$$
\hat{Q}=\int_{V(t)} Q d V \quad \text { and } \quad \hat{R}=\oint_{S(t)} \mathbf{H} \cdot \hat{n} d S .
$$

The $V$ is the volume and $S$ is the surface of the control volume. The $\hat{n}$ is the surface normal vector. The $\hat{S}$ is the axisymmetric source term

$$
\hat{S}^{T}=S_{\theta}(0,0, p, 0)
$$

which accounts for the surface integrals for the circumferential surfaces of an axisymmetric control volume. The $S_{\theta}$ is the area of the circumferential plane. The $p$ is the static pressure. For two-dimensional and three-dimensional flows, $\hat{S}$ is the zero vector. The $Q$ is the algebraic vector of conservative variables,

$$
Q^{T}=\left(\rho, \rho \vec{V}, E_{t}\right)
$$

where $\rho$ is the density, $\vec{V}=u \hat{\imath}+v \hat{\jmath}+w \hat{k}$, and $u, v$, and $w$ are the flow velocity components, respectively. The $E_{t}$ is the total energy per unit volume,

$$
E_{t}=\frac{p}{\gamma-1}+\frac{1}{2} \rho \vec{V} \cdot \vec{V}
$$

The $\mathbf{H}$ is the flux dyadic, which for a mixed EulerianLagrangian description ${ }^{4}$ is,

$$
\mathbf{H}=\mathbf{F}-\vec{g} Q \text {. }
$$

The $\vec{g}$ is the velocity vector of the control surfaces, $\vec{g}=x_{t} \hat{\imath}+y_{t} \hat{\jmath}+z_{t} \hat{k}$. An Eulerian description is obtained for $\vec{g}=0$ while a Lagrangian description is obtained for $\vec{g}=\vec{V}$. The $\mathbf{F}$ is the Cartesian flux dyadic.

One can express the integral form of Eq.(1) in a differential form,

$$
\frac{\partial Q}{\partial t}+\nabla \cdot \mathbf{F}=0
$$

which represents the fluid at a point and no longer contains direct information of the moving flow domain. The next section will discuss the coordinate transformations that will reintroduce the grid velocity terms to account for the boundary motion.

The flow model is complete with Sutherland's formula, the definition of the Prandtl number, and a perfect gas assumption. Turbulence models solve for the turbulent eddy viscosity. No modifications were made to the turbulence models of NPARC v3.0 to account for the effects of the moving grids on the convection of turbulent quantities. The significance of neglecting the convection of turbulent quantities due to the grid motion when using the one- or twoequation models has not been investigated in this work. The specification of boundary conditions and an initial solution then close the system of equations.

\section{Computational Methods}

The flow equations as presented in Eq.(7) are now discretized upon a structured grid using a finitedifference approximation. Terms due to the grid motion appear. NPARC solves the flow equations using a time-marching approach which starts with a known grid and solution. For unsteady flow simulation, a time step, which must be uniform over all the grid points, is determined and the flow equations are integrated to the new time level. For moving grids, the grid motion is defined such that the grid at the new time level is known, thus the grid velocities can be computed from a time difference over the time step interval. The modifications to the computational methods of NPARC version 3.0 required to account for moving grids are discussed in this section.

\section{Finite-Difference Form}

Eq.(7) is transformed from the physical coordinate system $(t, x, y, z)$ to a computational coordinate system $(\tau, \xi, \eta, \zeta)$ to allow for the body-conforming coordinate system used in NPARC. The transformation is defined such that $\tau=t$. The transformed equation can be put into strong conservation law form in which

$$
\hat{Q}=Q / J \quad \text { and } \quad \hat{R}=\frac{\partial \hat{F}_{j}}{\partial \xi_{j}} .
$$

where the generalized flux components for a timevarying grid are

$$
\hat{F}_{j}=\frac{1}{J}\left(\frac{\partial \xi_{j}}{\partial t} Q+\frac{\partial \xi_{j}}{\partial x_{k}} F_{k}\right)
$$


where the generalized and physical coordinates are $\xi_{j} \in(\xi, \eta, \zeta)$ and $x_{j} \in(x, y, z)$, respectively.

The $\partial \xi_{j} / \partial t$ are the time metrics of the transformation and are evaluated as

$$
\begin{aligned}
& \xi_{t}=-x_{\tau} \xi_{x}-y_{\tau} \xi_{y}-z_{\tau} \xi_{z} \\
& \eta_{t}=-x_{\tau} \eta_{x}-y_{\tau} \eta_{y}-z_{\tau} \eta_{z}
\end{aligned}
$$

and

$$
\zeta_{t}=-x_{\tau} \zeta_{x}-y_{\tau} \zeta_{y}-z_{\tau} \zeta_{z}
$$

where $\left(\xi_{x}, \xi_{y}, \xi_{z}, \eta_{x}, \eta_{y}, \eta_{z}, \zeta_{x}, \zeta_{y}, \zeta_{z}\right)$ are the spatial metrics and are defined in Ref. 2. For a planar domain, the time metrics reduce to

$$
\xi_{t}=-x_{\tau} \xi_{x}-y_{\tau} \xi_{y}
$$

and

$$
\eta_{t}=-x_{\tau} \eta_{x}-y_{\tau} \eta_{y}
$$

Within NPARC, the finite-difference representation of the above equations are approximated spatially through the use of a second-order, centraldifference. The viscous fluxes are evaluated explicitly using a second-order, central-difference. Second and fourth-order artificial dissipation are used to stabilize the flux computation. Further details on the methods used in NPARC are presented in Ref. 2.

\section{Newton Iterative Method}

NPARC v3.0 used a penta-diagonalized approximate factorization of the Euler implicit time difference to efficiently converge to a steady-state solution. NPARC v3.1 introduces a three-level, backwards time difference with a Newton iterative method to provide for nominally second-order time accuracy. Such a procedure has been presented in several references ${ }^{5,6,7}$. The iterative equation assuming a constant time step is

$$
\begin{aligned}
& {\left[I+\frac{2 \Delta \tau}{3} \frac{\partial \hat{A}^{m}}{\partial \xi_{j}}\right] \Delta \hat{Q}^{m}=} \\
& -\frac{2 \Delta \tau}{3}\left(\hat{R}^{m}-\hat{S}^{m}\right) \\
& -\left(\hat{Q}^{m}-\frac{4}{3} \hat{Q}^{n}+\frac{1}{3} \hat{Q}^{n-1}\right)
\end{aligned}
$$

where $\hat{A}$ is the Jacobian of $\hat{F}$ and $m$ is the subiteration index. Within NPARC v3.1, the effects of non-constant time steps are included. The Newton iterative method takes advantage of the existing NPARC solver with only minor modifications. Eq.(15) is iterated at each time step until the righthand-side, which is the discrete form of the flow equations, essentially becomes zero, which assures that Eq.(1) is essentially satisfied at each time step. This generally requires only a few sub-iterations at each time step since the initial solution is usually close to the solution at the new time. Nominally second-order time-accuracy is obtained through the sub-iterations even though the diagonalization is not second-order time-accurate. Further time-accuracy issues are discussed by Pulliam ${ }^{5}$.

The Newton iterative method is used in NPARC v3.1 when ISOLVE $=2$. A new input variable USUBMX indicates the maximum number of subiterations that are allowed at each time step. The input variable TOLSUB indicates the maximum tolerance on the iteration residual (displayed as L2 RESIDUAL in the output) at which the sub-iterations would cease.

The sub-iterations are performed on a block level, and so, information at the block boundary lags in time by at least one time step. Therefore, time accuracy will decrease at the block boundaries and it is suggested that block boundaries not be placed in regions where the time resolution of unsteady flow is desired.

\section{Explicit, Multi-Stage Methods}

The explicit, multi-stage methods of NPARC v3.0 can be used for second-order, time-accurate computations with moving grids. These methods are used when ISOLVE $=3,4$, or 5. However, the CFL condition limits the stable time step size, which was the reason the Newton iterative method was added in NPARC v3.1.

\section{Flow Boundary Conditions}

For NPARC, the boundary conditions are imposed explicitly. Temporal errors are reduced at the boundaries through the multi-stage procedures of the explicit methods and the iterative procedures of the Newton iterative method.

The flow boundary condition at the solid wall is the primary mechanism through which the flow senses that a boundary is in motion. The inclusion of the grid velocity vector $\vec{g}$ accounts for the motion of the boundary. The single physical flow boundary condition for a slip solid wall is

$$
\rho(\vec{V}-\vec{g}) \cdot \hat{n}=0
$$

which states that the component of the fluid velocity in the direction of the surface normal vector $\hat{n}$ must match the component of the grid velocity vector in the direction of the surface normal vector. An additional physical boundary condition that exists with 
the case of a no slip wall is

$$
\rho(\vec{V}-\vec{g}) \cdot \hat{t}=0
$$

which with Eq.(16) results in the condition

$$
\vec{V}=\vec{g}
$$

For other boundaries which may be in motion, the flow boundary conditions are modified by using the velocity at the boundary relative to the moving boundary. Thus, the convective velocity of interest is that relative to the moving boundary.

\section{Geometric Conservation Law}

The deformation of the grid is a source of possible errors. One must require that a uniform flow be preserved independent of the grid motion. This is stated in the geometric conservation $\mathrm{law}^{8}$ (GCL) which essentially relates the change in volume of the cell to the motion of the cell faces,

$$
\frac{d V}{d t}=\oint_{S(t)} \vec{g} \cdot \hat{n} d S .
$$

The geometric conservation law for the finitedifference form of Eq.(1) is

$$
\left(J^{-1}\right)_{\tau}=\left(\xi_{\tau} / J\right)_{\xi}+\left(\eta_{\tau} / J\right)_{\eta}+\left(\zeta_{\tau} / J\right)_{\zeta} .
$$

Applied to the implicit, Newton iterative method, the GCL can be used to find the Jacobian which yields uniform flow

$$
\begin{array}{r}
\left(J^{-1}\right)_{G C L}^{n+1}=\frac{4}{3}\left(J^{-1}\right)^{n} \\
-\frac{1}{3}\left(J^{-1}\right)^{n-1}+\frac{2 \Delta \tau}{3}\left(J^{-1}\right)_{\tau}^{n+1} .
\end{array}
$$

The term $\left(J^{-1}\right)_{T}^{n+1}$ is obtained by evaluating Eq.(20) using a second-order central-difference. The equation is an explicit expression because the grid and grid velocity are known for the new time level $\tau^{n+1}$.

At each time-level, the new value of $\hat{Q}$ can be evaluated as

$$
\hat{Q}^{n+1}=\hat{Q}^{n+1} J_{G C L}^{n+1} / J_{G R I D}^{n+1}
$$

where $J_{G R I D}^{n+1}$ is the Jacobian as computed from the known grid at time level $(n+1)$. The new solution is then decoded as

$$
Q^{n+1}=\hat{Q}^{n+1} J_{G R I D}^{n+1}
$$

For the Euler implicit method, the Jacobian for the geometric conservation law is determined from

$$
\left(J^{-1}\right)_{G C L}^{n+1}=\left(J^{-1}\right)^{n}-\Delta \tau\left(J^{-1}\right)_{T}^{n+1} \text {. }
$$

For the explicit, multi-stage methods, the Jacobian for the geometric conservation law is determined from

$$
\left(J^{-1}\right)_{G C L}^{n+1}=\left(J^{-1}\right)^{n}-\Delta \tau\left(J^{-1}\right)_{\tau}^{n}
$$

\section{Time Control and Step Size}

NPARC v3.0 uses local time step sizes to enhance convergence to steady-state; however, an unsteady flow simulation requires a spatially uniform time step size. The option IVARDT $=0$ allows a constant and uniform time step size to be input through the variable DTCAP; however, the time step size can not adjust to unsteady flow conditions. The option IVARDT = 4 allows adjustment; however, it does not assure that the time step size is uniform across blocks.

NPARC v3.1 adds several options for specifying the uniform time step size across all the blocks. The option IVARDT $=5$ examines all the blocks and picks out the minimum time step size based on the CFL condition and specified CFL number input through the variable DTCAP,

$$
\Delta \tau=\frac{\text { DTCAP }}{\sqrt{\left.\lambda_{\text {acoustic }}\right|_{\max }}}
$$

where

$$
\lambda_{\text {acoustic }}=|U|+a s
$$

where $U$ is the contravariant velocity, $s$ is the $L_{2}$ norm of the metrics. For the $\xi$-direction, these are

$$
U=\xi_{t}+\xi_{x} u+\xi_{y} v+\xi_{z} w
$$

and

$$
s=\left(\xi_{x}^{2}+\xi_{y}^{2}+\xi_{z}^{2}\right)^{1 / 2} .
$$

Similar expressions can be applied in the $\eta$ and $\zeta$ directions.

The option IVARDT $=6$ considers only the convective portion of the eigenvalues when computing the time step size using the CFL condition,

$$
\Delta \tau_{(J, K, L)}=1 / \max (|U|,|V|,|W|) .
$$

This restriction simply limits the time step size such that a fluid particle will not traverse a distance greater than the length of the cell. Thus, the overall time step size is limited by time accuracy considerations rather than the numerical stability.

NPARC v3.1 allows the simulation start time, TSTART, and end time, TFINAL, to be specified in the input file. For an unsteady flow simulation, the physical time is displayed to the standard output unit. When this time reaches TFIMAL, the computation will stop, unless the maximum number of time 
steps as specified by the input variable NMaX has been reached first.

\section{Grid Speeds}

The grid velocity vector is computed from a firstorder, forward time difference of the grids at two consecutive time levels

$$
\vec{g}=\left(\vec{r}^{n+1}-\vec{r}^{n}\right) / \Delta \tau
$$

where $\vec{r}$ is the position vector for the grid points.

\section{Restart File}

The initial grid and solution are input to NPARC through the restart file (fort.2). A new format for the restart file is introduced in NPARC v3.1 to allow the current non-dimensional time and time step to be listed in the first line. Also, the grid velocities can be specified for problems involving the rigid-body motion of the grid when IGRDYN = 1. A solution from the previous time step can also be included, which is needed when restarting a computation using the Newton iterative method. NPARC v3.1 can automatically detect which format is used. The input variable IRSTFL forces the restart file to be written using the new format. Further details on the new format can be found in the NPARC v3.1 user's guide $^{1}$.

\section{Moving Grids}

The previous sections discussed how the grid velocities are used within NPARC v3.1. This section discusses the details of the approach for specifying the grid dynamics problem and the methods for determining the grid distribution at each time level.

\section{Grid Motion}

The approach involves starting with the grid as read in from the restart file and defining the motion of that grid. Several types of grid motion are possible and the input variable IGRDYH indicates the type of motion of the grid:

A value of IGRDYN $=0$, which is the default, indicates that the grid should remain stationary throughout the computation. The grid velocities are set to zero and no grid regeneration is performed.

A value of IGRDYN $=1$ indicates that the entire grid can undergo rigid-body translation and / or rotation depending on the values of the grid velocities as read in from the restart file. The grid velocities are the absolute velocity components at each grid point as determined from rigid-body dynamics. The values of the grid velocities remain constant throughout the computation and no grid regeneration is performed.

A value of IGRDYN $=2$ indicates that one or more segments of the boundary grid translate, rotate, or deform relative to the rest of the grid. The parameters defining the dynamics of these segments are specified through the grid dynamics input file (fort.54). The grid becomes an explicit function of time. The grid is regenerated to obtain the grid at the new time level, $\vec{r}^{n+1}$, if there is boundary motion over the current time step. The grid speeds are computed from a time difference of the two grids. Two types of relative motion are possible. The first type is when a segment translates and rotates as a rigidbody relative to the rest of the boundary grid. The second type is when the segment deforms relative to itself and the rest of the boundary grid.

\section{Grid Dynamics Input File (fort .54)}

When IGRDYN $=2$, NPARC $\mathrm{v} 3.1$ reads file fort. 54 for the input parameters defining the dynamics of the moving boundary grid segments. The grid as read in through the restart file is taken as the state of the grid at the time specified in the restart file. The initial grid velocities may also read in from the restart file. The dynamics of the grid is defined by specifying which segments of the boundary grid are in motion, the type of motion, and time schedules for the motion. The segments are specified by listing for each segment the block and $(j, k)$ range. The segment must be along a constant $j$ or $k$ line and on the boundary. The segment may be one of three types:

Rigid. The segment translates and rotates about a defined rotation point. The additional inputs required are a rotation point and a schedule defining the translation and rotation with respect to time.

Variable. This segment can change shape to adjust the domain boundary to the motions of the rigidbody segments. The variable segment is defined by a cubic-spline using end position and tangent conditions. The additional inputs required include the type of end tangency conditions. The options include that the ends are not tangent, the ends remain tangent to the original segment, the ends remain tangent to the adjacent segments, or the ends remain normal to the adjacent segments (which is useful at intersections of block faces).

Deforming. This segment can deform according to a coded relation in the subroutine deform.f. Within NPARC v3.1 the subroutine deform. $f$ is coded to solve the test case of a flexible bump collapsing in a 
annular duct as described in a section below. One can code a different deformation into deform.f and then rebuild the NPARC executable.

One can refer to Ref. 1 for detailed information on form of the grid dynamics input file. As an example, consider Fig. 4 which shows the inviscid grid for the flap deflection problem discussed below. Three dynamic segments were defined: a rigid segment for the flap which rotates about the coordinate $(0.6,0.0)$ and two variable segments. The first variable segment attaches the flap to the forward portion of the flat plate. As the flap rotates, the variable segment provides a smooth connecting surface. The second variable segment is the outflow boundary which moves to accommodate the flap rotation in such a manner that the grid quality is maintained.

\section{Grid Regeneration}

When ISOLVE $=2$ and there exists some motion of the boundary segments over the time step, the grid is possibly deformed at each time step and must be regenerated at each time step. NPARC v3.1 includes an automated procedure to determine if any of the segments are in motion and regenerate the grid. The grid is regenerated using an efficient, algebraic, transfinite interpolation method and is only regenerated in those regions of the grid block with moving segments. This reduces the computational effort. For three-dimensional grids, it is required that the grid be "quasi-2d", which is specified by the input variable IGRDAX $=0$, or axisymmetric, which is specified by IGRDAX = 1 , within those blocks with moving boundaries. Thus, the boundary motion and grid regeneration remains a planar grid problem.

\section{Output Options}

Several options exist for providing output when there are moving grids. First, one can set ISOLVE $=0$ to perform the time-marching without computing the solution, but still create a time-varying grid. This allows one to check that the time-varying grid is being generated properly prior to beginning the flow computation. The output option HQFREQ allows grid and solution files to be written using the Plot3D file format at intervals of time steps. The file names are u\#\#\#. dat and u\#\#q. dat where \#\# is an index indicating the sequence number. These files may be used to observe the time history of the grid and solution and perhaps be part of an animation.

\section{Test Cases}

Several test cases involving moving grids are now presented to demonstrate the application and accu- racy of NPARC v3.1. All of the results were generated using a three-dimensional grid and NPARC3D v3.1. Unless specified otherwise, the computations used the Newton iterative method. Further discussion of these test cases and others can be found in the NPARC v3.1 user's guide ${ }^{l}$.

\section{Flying Wedge}

This test case involves a wedge of a half-angle of 15 degrees in an inviscid flow of Mach 2.5 relative to the wedge. The pressure ratio across the oblique shock and the Mach number behind the oblique shock are obtained from oblique shock theory ${ }^{9}$ and are presented in the first row of Table 1. Applying the Galilean transformation, one can view this problem as a stationary wedge in the absolute frame of reference with a Mach 2.5 flow past the wedge or as a moving wedge flying at Mach 2.5 through stationary air. Using the later view with NPARC v3.1 allows a demonstration of the ability to specify the grid velocities through the restart file and model a rigid-body motion of the entire grid. Computations were performed for both views using the Euler implicit method (ISOLVE=1) and local time stepping since only the steady-state was of interest. Fig. 1 shows the grid for the flow domain in which the leading edge of the wedge is at $x=1.0$.

Table 1. The results for a 15 degree wedge in Mach 2.5 flow.

\begin{tabular}{lccc}
\hline \hline & $p / p_{\infty}$ & Mach & \%Error \\
\hline Theory & 2.468 & 1.874 & - \\
Stationary Wedge & 2.470 & 1.896 & 1.17 \\
Flying Wedge & 2.470 & 1.897 & 1.23 \\
Inviscid Flap & 2.470 & 1.907 & 1.76 \\
Turbulent Flap & 2.485 & 1.893 & 1.03 \\
\hline \hline
\end{tabular}

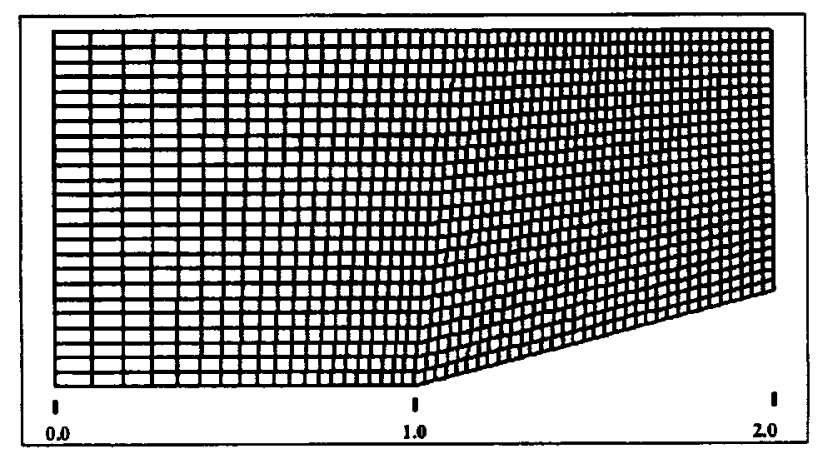

Figure 1: The grid for the 15 degree wedge.

The results of the stationary wedge case are presented in row 2 of Table 1 . The pressure ratio shows excellent agreement with theory. The third column 
of Table 1 presents the percentage error between the computed and theoretical Mach number. Since the pressure ratio for this and the other cases compare very well, their errors are not reported.

For the flying wedge case, the grid velocities are specified as Mach 2.5 and remained constant throughout the computation. The input variable IGRDYN = 1 and the initial restart file is created using the new format containing the grid velocities. Fig. 2 shows the Mach number contours of the flowfield to provide an overall picture of the computational results.

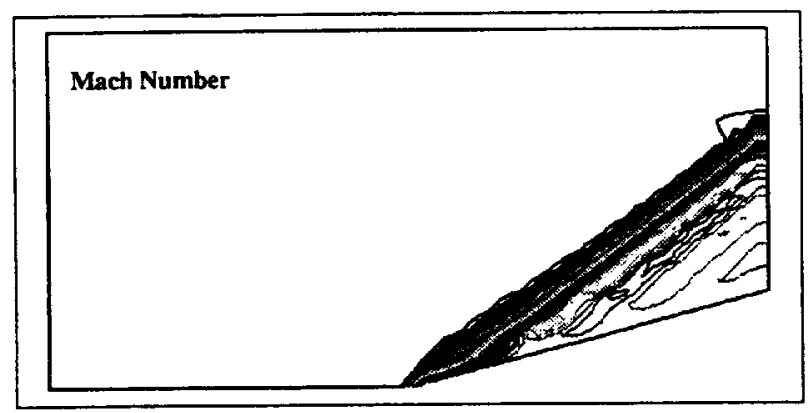

Figure 2: The Mach number contours for the flying 15 degree wedge.

The computed pressure and density distributions along surface of the wedge are identical for both the stationary and flying wedge cases. However, Fig. 3 shows a significant difference in the Mach number distribution due to the fact that for the flying wedge problem, the velocities on the wedge surface are imposed as part of the boundary condition. Table 1 shows good agreement of the pressure ratios and Mach numbers behind the shock between the stationary and flying wedge cases.

\section{Flap}

A simple case involving the relative motion of a segment of the boundary grid is the rotation of a flap on a flat plate through an angle of 15 degrees in a flow of Mach 2.5. Fig. 4 shows the outline of the flow domain of the flat plate prior to rotation and the grid after rotation. The flat plate is 1.2 units long with the aft 0.5 units being the flap. The flap rotates within 1.0 nondimensional time units and is specified to rotate about a fixed rotation of $(0.6,0.0)$ units.

Once the flap has rotated, the flow approaches a steady-state flow with an oblique shock of the same strength as the wedge problem above and same analytic solution. The flap segment is defined to be a rigid segment with the indicated rotation and rotation point. Two variable segments are defined to

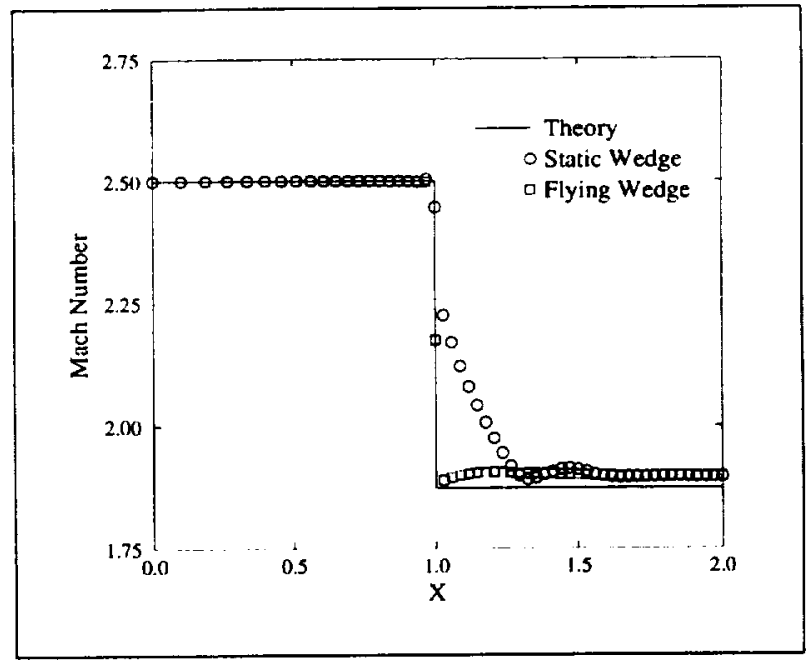

Figure 3: The variation of the Mach number along the surface of a 15 degree wedge.

attach the flap to the flat plate and to allow the outflow boundary to deform with the flap motion, respectively, and are identified in Fig. 4. For the computation, IGRDYN $=2$ and IVARDT $=5$. Both inviscid case and turbulent flow case, which used the Baldwin-Lomax turbulence model, were computed. Fig. 5 shows the Mach number contours for the inviscid case flowfield to provide an overall view of the flowfield. Table 1 shows the computed the pressure ratio and Mach number behind the oblique shock for the steady-state solutions, which compare very well with theory.

\section{Collapsing Bump in a Duct}

This case demonstrates the ability of NPARC v3.1 to solve the unsteady flow with a deforming boundary segment. An axisymmetric bump on the hub of an annular duct collapses within $0.8 \mathrm{milli}-$ seconds (msec) in stationary flow conditions. Once collapsed, the bump forms a cylindrical section flush with the hub. Fig. 6 shows the grid near the bump for several times during the bump collapse.

The bump collapse creates acoustic disturbances which propagate away from the bump and towards the ends of the annular duct. Fig. 7 shows the pressure contours at several instances in time with the initial stationary flowfield at the left of the figure. The pressure contours near the bump collapse show both radial and axial variations; however as the acoustic disturbances propagate toward the end of the duct, the disturbances obtain a planar character.

This case was the product of experiments conducted at the University of Cincinnati ${ }^{10}$ and the subject of detailed computational analysis in Ref. 


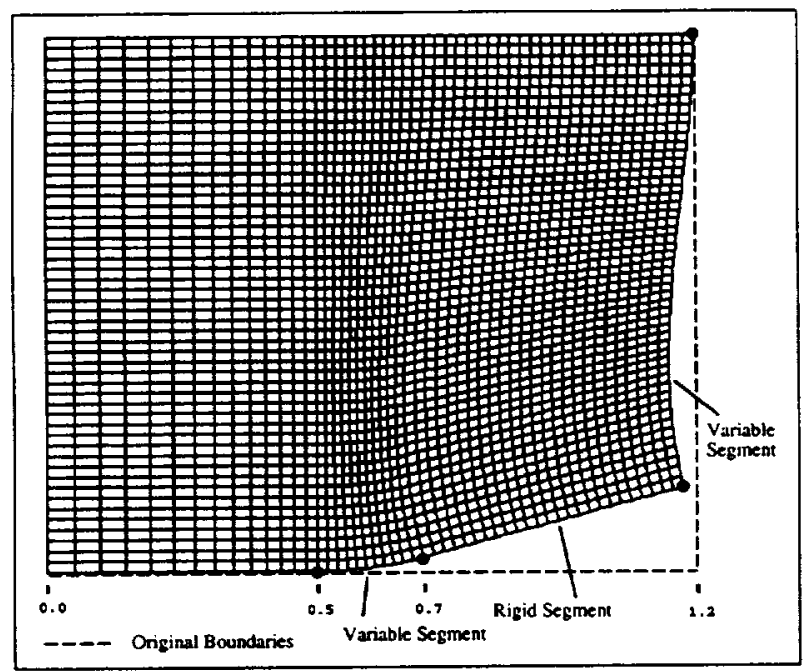

Figure 4: The grid for the inviscid case for the flap at 15 degree rotation in Mach 2.5 flow.

7. The experiment provided the time variation of the pressure at a few sensor locations along the duct. Fig. 8 shows the comparison between the computed and experiment unsteady pressures at one of those sensor locations. This case demonstrates the application of NPARC v3.1 for solving problems with deforming boundary grid segments with IGRDYN $=2$. The three-dimensional flowfield was computed with the assumption of an axisymmetric, three-dimensional grid using the input variable IGRDAX = 1. The grid dynamics input file included one deforming segment. The deformation was computed using the relations coded into the deform subroutine of the dgmod.f file of NPARC v3.1.

\section{High-Speed Inlet Restart}

A more complex test case involving multiple grid blocks is the unstart / restart operation of the NASA variable diameter centerbody (VDC) inlet ${ }^{11}$. The NASA VDC inlet is an axisymmetric, mixedcompression inlet. In normal cruise operations, the Mach 2.5 freestream flow is compressed both exterior and interior to the inlet and a normal shock is positioned just aft of the throat. The analysis here assumes inviscid flow.

The steady-state, supercritical flowfield was first computed using the Euler implicit method (ISOLVE =1) with local time stepping. The Chung-Cole compressor face boundary condition ${ }^{6}($ BCTYPE $=4$ ), which was also introduced in NPARC v3.1, was applied with a compressor face Mach number of Mach 0.3 .

The unstart was initiated by a $5 \%$ decrease in the compressor-face Mach number, which reduces to

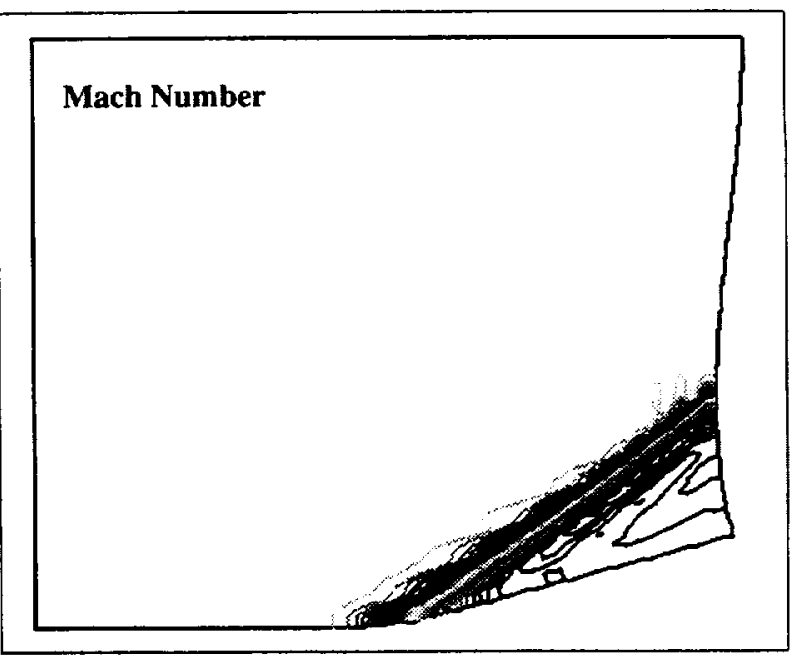

Figure 5: The Mach number contours for the flap at 15 degree rotation in Mach 2.5 flow.

a value of Mach 0.285 . The normal shock is pushed forward past the throat and is expelled from the inlet.

The restart operation is initiated by increasing the Mach number at the compressor face to a value of Mach 0.5, which simulates the opening of bypass doors. The inlet geometry is varied by translating the centerbody forward 0.2 units and rotating the forebody cone from 18.5 degrees to 12.5 degrees to increase the throat area. The shock moves downstream and is reformed within the subsonic diffuser of the inlet. Once a normal shock is again formed in the diffuser, the inlet is returned to its cruise configuration.

The three-dimensional flowfield was computed with the assumption of an axisymmetric, th.reedimensional grid using the input variable IGRDAX = 1. The grid dynamics input file included three rigid segments for the nose cone, forebody cone, and aft cone. The nose cone simply translated while the forebody and aft cones also rotated about their respective rotation points. Three variable segments were defined to connect the nose cone to the inflow boundary, connect the forebody cone to the aft cone (which physically is the bleed slot region), and connect the aft cone to the compressor face boundary. Fig. 9 shows the extent of the grid motion by showing the grid near the entrance of the inlet at the cruise configuration and at the restart configuration with the centerbody translated and forebody cones rotated. The bleed slot region is identified to better see the extent of the translation and the increase in the throat area due to the rotation of the forebody and aft cones. Note that the grid regeneration method retains good grid quality. The grid consists 


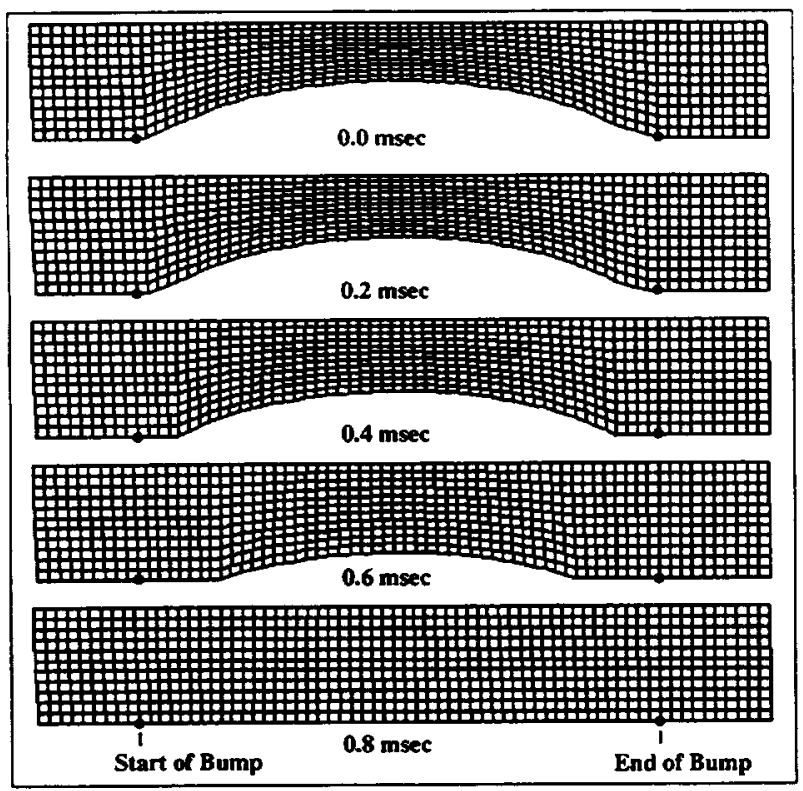

Figure 6: The time history of the grid through the bump collapse (top-bottom: $t=0.0 \mathrm{msec}$, $t=0.2 \mathrm{msec}, t=0.4 \mathrm{msec}, t=0.6 \mathrm{msec}, t=0.8$ msec).

of three blocks with block 1 extending axially along the centerbody and the interior of the cowl. Blocks 2 and 3 form the exterior flow area and do not involve any grid motion.

Fig. 10 shows a series of Mach number contours during the unstart / restart operation to provide an overall view of the unsteady flowfield. The movement of the shock wave structure can be seen. The centerbody is collapsed between the time from $t=30$ msec to the time of $t=51 \mathrm{msec}$. Thus in Fig. 10, the centerbody is fully collapsed at a time of $t=4.0$ msec. The centerbody geometry variation presented in Fig. 10 occurs much faster than would occur in the actual inlet. This was done for computational convenience. However, the unsteady motion of the shocks is reasonable. There is no experiment data available for comparison; however, the results seem reasonable and provide a demonstration of the moving grid capabilities of NPARC v3.1.

\section{Summary and Conclusions}

A moving grid capability for NPARC has been introduced in version 3.1 and can be used to simulate the unsteady flow on a moving multi-block, structured grid with second-order time accuracy. It is assumed that a three-dimensional grid is "quasi-2d" or axisymmetric in the moving grid regions. The modifications to NPARC v3.0 to account for the moving grid and the approach for defining the moving grid problem was discussed. Simple test cases involving supersonic flow over a wedge and flow about a rotating flap showed good comparison with oblique shock theory. A test case involving the collapse of an axisymmetric bump in an annular duct showed excellent agreement with experiment data. The unstart / restart operation of the NASA variable diameter centerbody (VDC) inlet, which involved the translation and collapse of the centerbody, was demonstrated to show qualitative agreement with experimentally observed behavior.

\section{Acknowledgements}

The author would like to thank Dr. Joongkee Chung of the Institute for Computational Methods in Propulsion (ICOMP) at the Ohio Aerospace Institute and Scott Townsend of NYMA, Inc. for their work in the development of NPARC v3.1. Further, the author would like to thank Charles Lawrence, Rich Blech, and Gary Cole of NASA Lewis for their support for the development of NPARC v3.1.

\section{References}

[1] J. Chung, J.W. Slater, A. Suresh, and S. Townsend, "NPARC v3.1 User's Guide: A Companion to the NPARC v3.0 User's Guide," October, 1997.

[2] Cooper, G.K. and Sirbaugh, J.R., "The PARC Code: Theory and Usage," AEDC-TR-89-15, December 1989.

[3] "NPARC Version 3.0 User's Manual", September 1996.

[4] Vinokur, M., "An Analysis of Finite-Difference and Finite-Volume Formulations of Conservation Laws," Journal of Computational Physics, Vol. 81, 1989, pp. 1-52.

[5] Pulliam, T.H., "Time Accuracy and the Use of Implicit Methods," AIAA Paper 93-3360, July 1993.

[6] Chung, Joongkee and G.L. Cole, "Comparison of Compressor Face Boundary Conditions for Unsteady CFD Simulations of Supersonic Inlet," AIAA Paper 95-2627, July 1995.

[7] Slater, J.W., "Study of CFD Methods Applied to Rapidly Deforming Boundaries," AIAA Paper 97-2041, June 1997. 
[8] Thomas, P.D. and Lombard, C.K., "Geometric Conservation Law and its Application to Flow Computations on Moving Grids," AIA A Journal, Vol. 17, No. 10, 1979, pp. 1030-1037.

[9] Anderson, J.D., Modern Compressible Flow, McGraw Hill Inc., New York, 1984.

[10] Freund, D.D., Sajben, M., and Slater, J.W., "Compressor-Face Boundary Condition Experiment: Generation of Acoustic Pulses in Annular Ducts," AIAA Paper 96-2657, July 1996.

[11] Wasserbauer, J. et al., "Design of a Very-LowBleed Mach 2.5 Mixed-Compression Inlet with 45 Percent Internal Contraction," NASA TM X-3135, 1974.

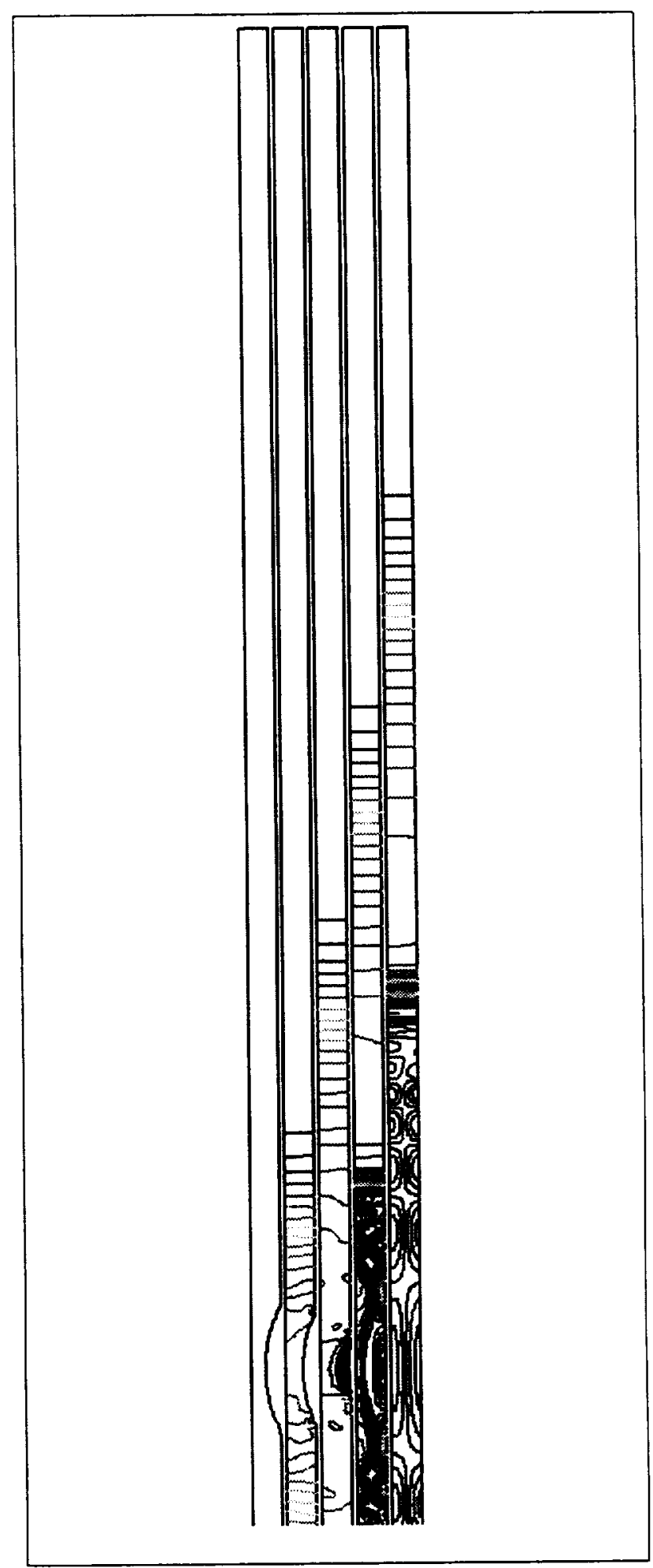

Figure 7: The pressure contours during and after the bump collapse (left-right: $t=0.0 \mathrm{msec}$, $t=0.4 \mathrm{msec}, t=0.8 \mathrm{msec}, t=1.2 \mathrm{msec}, t=1.6$ msec). 


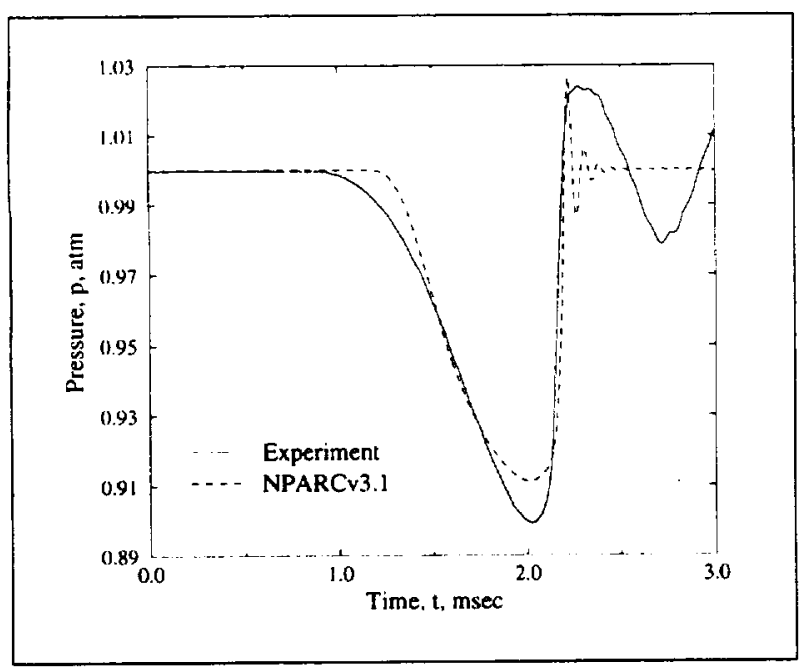

Figure 8: The comparison between the computed and experiment time history of the static pressure at the sensor location.

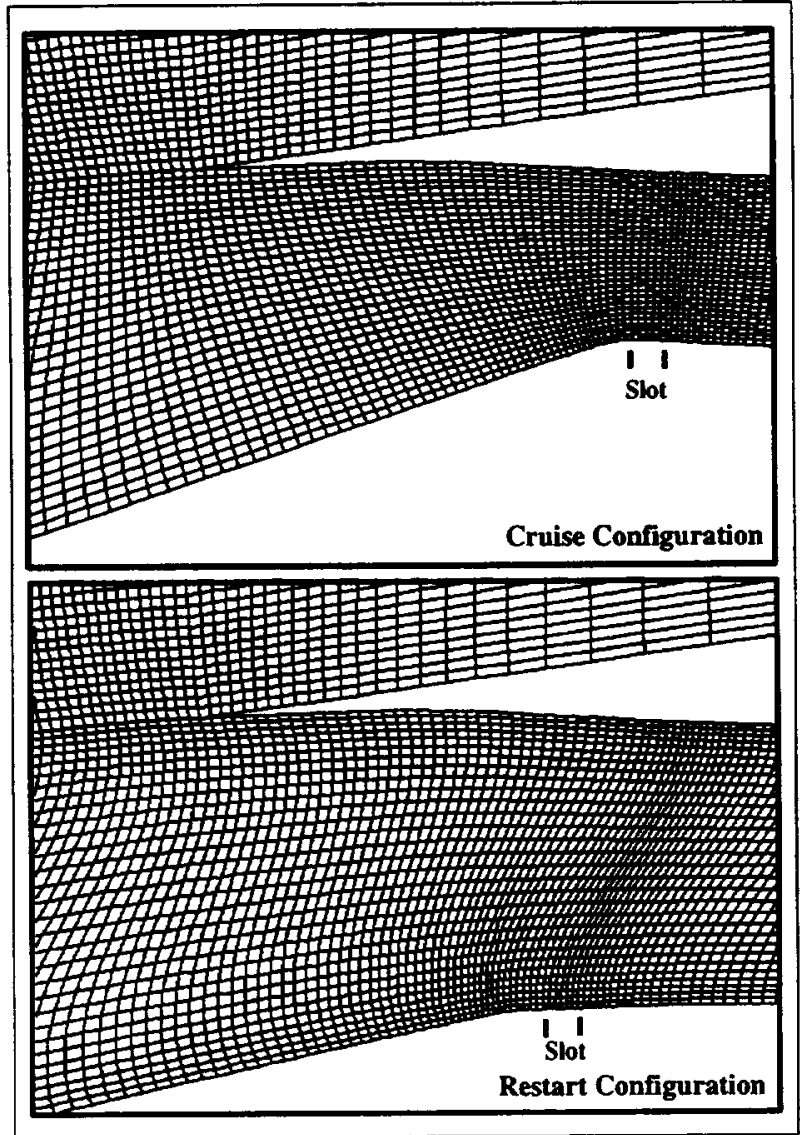

Figure 9: The grid for the NASA VDC inlet in the region of the inlet entrance for the cruise configuration (top) and the restart configuration (bottom) with the location of the bleed slot identified.
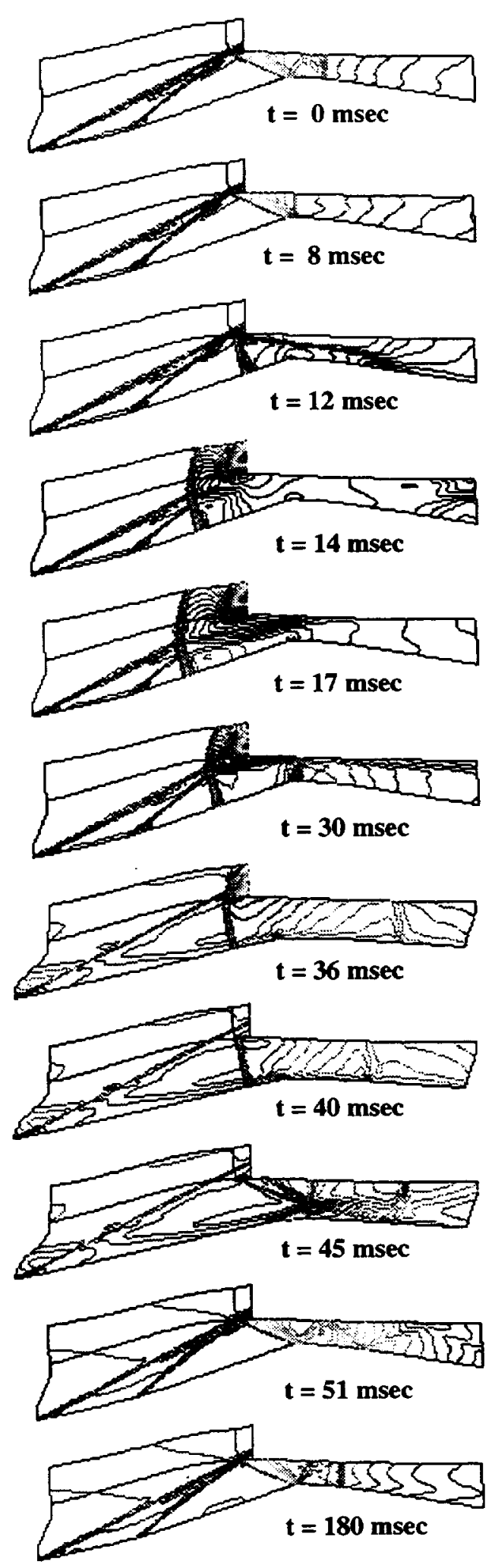

Figure 10: The Mach number contours for the unstart / restart operation of the NASA VDC inlet. 


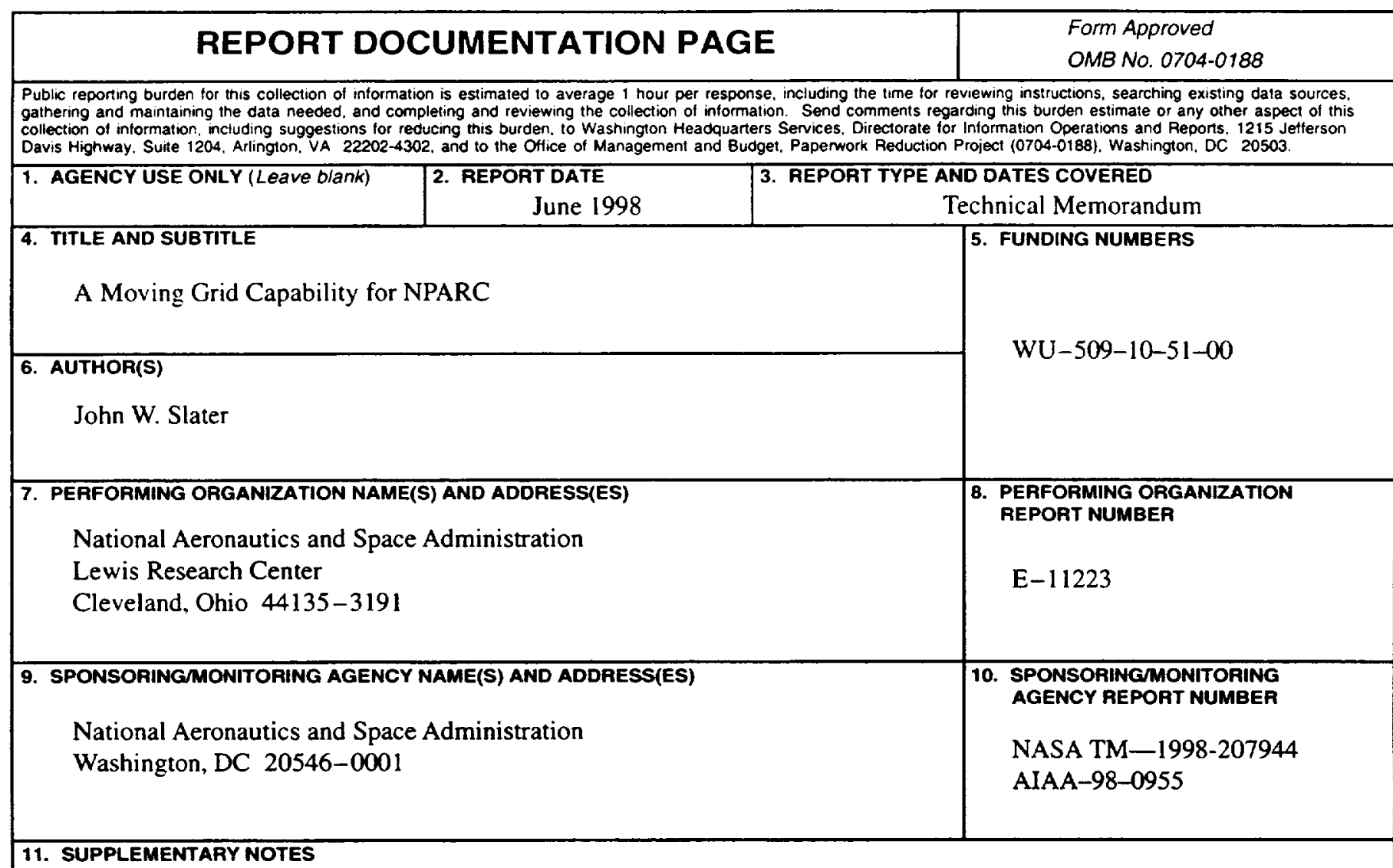

\section{SUPPLEMENTARY NOTES}

Prepared for the 36th Aerospace Sciences Meeting and Exhibit sponsored by the American Institute of Aeronautics and Astronautics, Reno, Nevada, January 12-15, 1998. Responsible person, John W. Slater, organization code 5850, (216) 433-8513.

\section{2a. DISTRIBUTIONAAVAILABILITY STATEMENT}

12b. DISTRIBUTION CODE

Unclassified - Unlimited

Subject Categories: 01 and 07

Distribution: Nonstandard

This publication is available from the NASA Center for AeroSpace Information, (301) 621-0390.

13. ABSTAACT (Maj-imum 200 words)

Version 3.1 of the NPARC computational fluid dynamics flow solver introduces a capability to solve unsteady flow on moving multi-block, structured grids with nominally second-order time accuracy. The grid motion is due to segments of the boundary grid that translate and rotate in a rigid-body manner or deform. The grid is regenerated at each time step to accommodate the boundary grid motion. The flow equations and computational models sense the moving grid through the grid velocities, which are computed from a time-difference of the grids at two consecutive time levels. For three-dimensional flow domains, it is assumed that the grid retains a planar character with respect to one coordinate. The application and accuracy of NPARC $\mathrm{v} 3.1$ is demonstrated for flow about a flying wedge, rotating flap, a collapsing bump in a duct, and the upstart / restart flow in a variable-geometry inlet. The results compare well with analytic and experimental results.

\section{SUBJECT TERMS}

Computational fluid dynamics; Unsteady flow; Moving geometry; Variable geometry

17. SECURITY CLASSIFICATION
OF REPORT
Unclassified

NSN 7540-01-280-5500

\begin{tabular}{|c|c|}
\hline $\begin{array}{c}\text { 18. SECURITY CLASSIFICATION } \\
\text { OF THIS PAGE } \\
\text { Unclassified }\end{array}$ & $\begin{array}{c}\text { 19. SECURTY CLASSIFICATION } \\
\text { OF ABSTRACT }\end{array}$ \\
Unclassified
\end{tabular}

